\title{
Make Things Now! \\ Pragmatic Functional Programming in Haskell
}

\author{
Don Stewart \\ Standard Chartered Bank \\ dons00@gmail.com
}

\begin{abstract}
For the past decade I've been building all kinds of software in Haskell: software for programming languages research; open source software as part of the Haskell.org project; and, more recently, commercial software for business and government.

This talk will look at the experience of delivering software written in Haskell, and how language features and tools can help you achieve a range of engineering goals. Beyond just technical issues though, we will look at how programmers that use Haskell and typed functional programming approach problems differently, and how small, skilled teams can do things faster and better.
\end{abstract}

